\title{
ON STRICHARTZ'S UNCERTAINTY INEQUALITY FOR THE HEISENBERG GROUP
}

\author{
Chettutty Smitha And Sundaram Thangavelu
}

(Received August 20, 2001, revised April 10, 2003)

\begin{abstract}
The aim of this article is to obtain a lower bound for the variance of a normalised $L^{2}$ function on the Heisenberg group under the assumption that its Fourier transform is small along a sequence of well distributed rays in the Heisenberg fan. This is achieved by proving an uncertainty inequality for Laguerre series which is analogous to the one obtained by Strichartz for spherical harmonic expansions. Applications to Hermite and special Hermite expansions are also given.
\end{abstract}

1. Introduction. The classical Heisenberg-Weyl uncertainty inequality for the Fourier transform on $\boldsymbol{R}^{n}$ says that

$$
\left(\int_{\boldsymbol{R}^{n}}|x|^{2}|f(x)|^{2} d x\right)\left(\int_{\boldsymbol{R}^{n}}|\xi|^{2}|\hat{f}(\xi)|^{2} d \xi\right) \geq \frac{n^{2}}{4}\left(\int_{\boldsymbol{R}^{n}}|f(x)|^{2} d x\right)^{2}
$$

This means that for a normalised $f \in L^{2}\left(\boldsymbol{R}^{n}\right)$ the variance of $|f(x)|^{2} d x$ has to be large if $\hat{f}(\xi)$ is small. There are many other situations where we have analogues of the Fourier transform but the perfect symmetry between the function and its Fourier transform is something very special to the Euclidean case. Rather than looking for a perfect analogue of (1.1) we can ask the question of finding suitable conditions on the Fourier transform which will ensure that the variance is large.

This point of view has been taken in Strichartz [5], where he has formulated and proved new uncertainty inequalities for the Euclidean Fourier transform and spherical harmonic expansion. For example, it was shown that for a normalised $f \in L^{2}(\boldsymbol{R})$,

$$
\int x^{2}|f(x)|^{2} d x \geq \frac{c \varepsilon^{2}}{b^{2}}
$$

if the Fourier transform is small in the sense that

$$
\left(\sum_{j=-\infty}^{\infty}\left|\hat{f}\left(a_{j}\right)\right|^{2}\right)^{1 / 2} \leq \frac{1-\varepsilon}{\sqrt{b}}
$$

where $a_{j}$ is a well distributed sequence with $a_{j+1}-a_{j} \leq b$. More interesting is the uncertainty inequality obtained for spherical harmonic expansions.

2000 Mathematics Subject Classification. Primary 42B10; Secondary 43A85.

Key words and phrases. Fourier transform, Hermite and Laguerre functions, spherical harmonics, Heisenberg group, representations. 
If $f_{k}$ are the spherical harmonic components of a normalised $f \in L^{2}\left(S^{n-1}\right)$, then Strichartz has obtained the lower bound

$$
\inf _{y \in S^{n-1}} \int_{S^{n-1}}|\sin d(x, y)|^{2}|f(x)|^{2} d x \geq c b^{-2}
$$

under a suitable smallness assumption on the components $f_{k}$. More precisely, let $\left\{a_{j}\right\}$ be an increasing sequence of even numbers and $\left\{b_{j}\right\}$ an increasing sequence of odd numbers such that $a_{j+1}-a_{j} \leq b, b_{j+1}-b_{j} \leq b, a_{0} \leq b$ and $b_{0} \leq b$. Then the lower bound (1.4) is valid whenever

$$
\sum_{j=0}^{\infty}\left\|f_{a_{j}}\right\|_{2}^{2}+\left\|f_{b_{j}}\right\|_{2}^{2} \leq c b^{-1}
$$

with $c$ sufficiently small.

In [5] Strichartz mentions several interesting open problems. One among them is to find an analogue of (1.4) for eigenfunction expansions associated to Schrodinger operators. In this article we prove analogues of (1.4) for Hermite, special Hermite and Laguerre expansions. A simple minded analogue of (1.2) can be proved for the Fourier transform on the Heisenberg group. By writing down the Plancherel theorem for the Heisenberg group in terms of certain representations of the Heisenberg motion group, we formulate an uncertainty inequality for the Fourier transform on the Heisenberg group.

There is a vast literature on various generalisations of the uncertainty principle. We refer the reader to the survey of Folland and Sitaram [1] and references given there.

The first author wishes to thank the Indian Academy of Sciences for the Summer Research Fellowship during which period this research was started. She also wishes to thank the Indian Statistical Institute for the hospitality and facilities provided during several visits. Both authors wish to thank Ms. Ashalata for her incredible efficiency in typing the manuscript. They also thank the referee for his careful reading of the manuscript and pointing out some errors in the previous version.

2. Hermite expansions. Normalised Hermite functions on the real line are defined by

$$
h_{k}(t)=\left(2^{k} k ! \sqrt{\pi}\right)^{-1 / 2} e^{t^{2} / 2} \frac{d^{k}}{d t^{k}}\left(e^{-t^{2}}\right) .
$$

The $n$-dimensional Hermite functions are then defined by taking tensor products. For each $\alpha \in \boldsymbol{N}^{n}$ and $x \in \boldsymbol{R}^{n}$, we define

$$
\Phi_{\alpha}(x)=\prod_{j=1}^{n} h_{\alpha_{j}}\left(x_{j}\right) .
$$

These functions $\left\{\Phi_{\alpha} ; \alpha \in \boldsymbol{N}^{n}\right\}$ form an orthonormal basis for $L^{2}\left(\boldsymbol{R}^{n}\right)$. Moreover, they are eigenfunctions of the Hermite operator $H=-\Delta+|x|^{2}$ as well as the Fourier transform. We refer to [6] for more information about Hermite functions and expansions in terms of them. 
Since $H \Phi_{\alpha}=(2|\alpha|+n) \Phi_{\alpha}$, the $k$-th eigenspace corresponding to the eigenvalue $(2 k+n)$ is spanned by $\left\{\Phi_{\alpha} ;|\alpha|=k\right\}$. Let $P_{k}$ be the orthogonal projection of $L^{2}\left(\boldsymbol{R}^{n}\right)$ onto the $k$-th eigenspace. Then $P_{k}$ is an integral operator whose kernel is

$$
\Phi_{k}(x, y)=\sum_{|\alpha|=k} \Phi_{\alpha}(x) \Phi_{\alpha}(y) .
$$

The Plancherel theorem for the Hermite expansions reads as $\|f\|_{2}^{2}=\sum_{k=0}^{\infty}\left\|P_{k} f\right\|_{2}^{2}$.

In this section we are interested in proving the following uncertainty inequality for the Hermite expansions. Given $A \subset N$, we define

$$
E_{A}(f)^{2}=\sum_{j \in A}\left\|P_{j} f\right\|_{2}^{2} .
$$

With this notation we have

THEOREM 2.1. Let $A$ be the union of two sequences $\left\{a_{j}\right\}$ and $\left\{b_{j}\right\}$, where $a_{j}$ are all even and $b_{j}$ odd and they satisfy $a_{j+1}-a_{j} \leq b, b_{j+1}-b_{j} \leq b$. Also, assume that $a_{0} \leq$ $b, b_{0} \leq b$. Let $f \in L^{2}\left(\boldsymbol{R}^{n}\right)$. Then

$$
\int_{\boldsymbol{R}^{n}}|x|^{2}|f(x)|^{2} d x \geq \frac{1}{8} b^{-2} \int_{\boldsymbol{R}^{n}}|f(x)|^{2} d x,
$$

whenever $E_{A}(f)^{2} \leq(1 / 4) b^{-1}\|f\|_{2}^{2}$ holds.

This theorem will be proved by reducing it to an uncertainty inequality for the Laguerre expansions. We pause for a moment to make the following observation. The above inequality can be thought of as a refinement of the Heisenberg-Weyl uncertainty inequality. Since $\Phi_{\alpha}$ are eigenfunctions of the Fourier transform with eigenvalue $(-i)^{|\alpha|}$, it follows that $P_{j} \hat{f}=$ $(-i)^{j} P_{j} f$ and therefore $E_{A}(f)=E_{A}(\hat{f})$. Thus for normalised functions $f \in L^{2}\left(\boldsymbol{R}^{n}\right)$ for which $E_{A}(f)^{2} \leq(1 / 4) b^{-1}$, each of the factors in (1.1) has the lower bound $(1 / 8) b^{-2}$.

Given $f \in L^{2}\left(\boldsymbol{R}^{n}\right)$, we consider the spherical harmonic expansion of $f$. Let $\mathcal{S}_{m}$ be the space of spherical harmonics of degree $m$. Writing $x=|x| x^{\prime}, x^{\prime} \in S^{n-1}$, we expand $f(x)=f\left(|x| x^{\prime}\right)$ in terms of spherical harmonics as $f(x)=\sum_{m=0}^{\infty} f_{m}(x)$ with $f_{m} \in \mathcal{S}_{m}$ when restricted to $S^{n-1}$. These $f_{m}$ can be expressed in terms of certain representations of the orthogonal group $O(n)$.

The natural action of $O(n)$ on $S^{n-1}$ defines a unitary representation on $L^{2}\left(S^{n-1}\right)$. The restrictions of this to each $\mathcal{S}_{m}$ defines an irreducible unitary representation denoted by $\delta_{m}$. Let $\chi_{m}$ be the character and $d_{m}$ the degree of $\delta_{m}$. Then we have

$$
f_{m}(x)=d_{m} \int_{O(n)} \chi_{m}(\sigma) f(\sigma \cdot x) d \sigma .
$$

A proof of this can be found in Helgason [3]. The kernels $\Phi_{k}(x, y)$ of the projections $P_{k}$ satisfy the property $\Phi_{k}(\sigma x, \sigma y)=\Phi_{k}(x, y)$ for every $\sigma \in O(n)$. This follows from the fact 
that $\Phi_{k}$ are given by the generating function

$$
\begin{aligned}
\sum_{k=0}^{\infty} r^{k} \Phi_{k}(x, y)= & \pi^{-n / 2}\left(1-r^{2}\right)^{-n / 2} \exp \left((-1 / 2)\left(\left(1+r^{2}\right) /\left(1-r^{2}\right)\right)\left(|x|^{2}+|y|^{2}\right)\right. \\
& \left.+\left(2 r /\left(1-r^{2}\right)\right) x \cdot y\right)
\end{aligned}
$$

valid for $|r|<1$.

In view of the above observations we infer that $P_{k} f_{m}=\left(P_{k} f\right)_{m}$. Since the spherical harmonic spaces $\mathcal{S}_{m}$ give an orthogonal decomposition of $L^{2}\left(S^{n-1}\right)$, we have $\|f\|_{2}^{2}=$ $\sum_{m=0}^{\infty}\left\|f_{m}\right\|_{2}^{2}$. Therefore,

$$
E_{A}(f)^{2}=\sum_{j \in A} \sum_{m=0}^{\infty}\left\|P_{j} f_{m}\right\|_{2}^{2}=\sum_{m=0}^{\infty} E_{A}\left(f_{m}\right)^{2} .
$$

Let $\left\{S_{m k} ; k=0,1,2, \ldots, d_{m}\right\}$ be an orthonormal basis for $\mathcal{S}_{m}$ so that

$$
f_{m}(x)=\sum_{k=1}^{d_{m}} f_{m k}(x)=\sum_{k=1}^{d_{m}} \tilde{f}_{m k}(|x|) S_{m k}\left(x^{\prime}\right) .
$$

Now, each piece $f_{m k}(x)=\tilde{f}_{m k}(|x|) S_{m k}\left(x^{\prime}\right)$ is left invariant by the Hermite projections. In fact, we have the following result.

Let $L_{k}^{\alpha}(t)$ be the Laguerre polynomials of type $\alpha>-1, k=0,1,2, \ldots$. Given a function $g \in L^{2}\left(\boldsymbol{R}^{+}, r^{2 \alpha+1} d r\right)$, we define

$$
R_{k}^{\alpha}(g)=2 \frac{\Gamma(k+1)}{\Gamma(k+\alpha+1)} \int_{0}^{\infty} g(r) L_{k}^{\alpha}\left(r^{2}\right) e^{-r^{2} / 2} r^{2 \alpha+1} d r .
$$

Functions of the form $P(x)=|x|^{m} S(x)$ where $S \in \mathcal{S}_{m}$ are called solid harmonics.

Proposition 2.2. Let $f(x)=g(|x|) P(x)$, where $P$ is a solid harmonic of degree $m$. Then $P_{2 k+m} f(x)=R_{k}^{\alpha}(g) P(x) L_{k}^{\alpha}\left(|x|^{2}\right) e^{-|x|^{2} / 2}$ where $\alpha=n / 2+m-1 . P_{j} f=0$ for all other values of $j$.

A proof of this proposition can be found in [6, Theorem 3.4.1]. From the proposition it follows that

$$
E_{A}(f)^{2}=\sum_{m=0}^{\infty} E_{A}\left(f_{m}\right)^{2}=\sum_{m=0}^{\infty} \sum_{k=1}^{d_{m}} E_{A}\left(f_{m k}\right)^{2} .
$$

A similar decomposition is true for $\|f\|_{2}^{2}$ as well as for $\int|x|^{2}|f(x)|^{2} d x$. In view of these remarks, in order to prove Theorem 2.1, it is enough to establish

$$
\int|x|^{2}\left|f_{m k}(x)\right|^{2} d x \geq(1 / 4) b^{-2} \int\left|f_{m k}(x)\right|^{2} d x-(1 / 2) b^{-1} E_{A}\left(f_{m k}\right)^{2} .
$$

Then, by adding all these inequalities, we obtain Theorem 2.1 .

By the proposition, all the integrals in the above inequality are expressible in terms of Laguerre coefficients. Thus everything boils down to establishing an uncertainty inequality for Laguerre series. This will be stated and proved in Section 5. 
3. Special Hermite expansions. In this section we are interested in proving an uncertainty inequality for expansions in terms of special Hermite functions which are eigenfunctions of the operator

$$
L=-\Delta+\frac{1}{4}|z|^{2}-i \sum_{j=1}^{n}\left(x_{j} \frac{\partial}{\partial_{y_{j}}}-y_{j} \frac{\partial}{\partial_{x_{j}}}\right) .
$$

For each pair of multiindices $(\alpha, \beta)$ we have the function on $\boldsymbol{C}^{n}$ defined by

$$
\Phi_{\alpha \beta}(z)=(2 \pi)^{-n / 2} \int_{\boldsymbol{R}^{n}} e^{i(x \cdot \xi+(1 / 2) x \cdot y)} \Phi_{\alpha}(\xi+y) \Phi_{\beta}(\xi) d \xi,
$$

where $z=x+i y$. These are eigenfunctions of $L$ with eigenvalues $(2|\beta|+n)$ and they form an orthonormal basis for $L^{2}\left(\boldsymbol{C}^{n}\right)$. For more about this expansion we refer to [6].

The special Hermite expansion of an $L^{2}$ function $f$ on $\boldsymbol{C}^{n}$ can be written in a compact form. Define

$$
\varphi_{k}(z)=L_{k}^{n-1}\left(\frac{1}{2}|z|^{2}\right) e^{-(1 / 4)|z|^{2}},
$$

where $L_{k}^{n-1}$ are Laguerre polynomials of type $(n-1)$. Let

$$
f \times g(z)=\int_{C^{n}} f(z-w) g(w) e^{(i / 2) \operatorname{Im}(z \cdot \bar{w})} d w
$$

be the twisted convolution of two functions $f$ and $g$. Then it can be shown that

$$
\sum_{|\beta|=k} \sum_{\alpha}\left(f, \Phi_{\alpha \beta}\right) \Phi_{\alpha \beta}(z)=(2 \pi)^{-n} f \times \varphi_{k}(z)
$$

so that the special Hermite expansion of $f$ reads

$$
f(z)=(2 \pi)^{-n} \sum_{k=0}^{\infty} f \times \varphi_{k}(z) .
$$

Note that $(2 \pi)^{-n} f \times \varphi_{k}(z)$ is the orthogonal projection of $L^{2}\left(\boldsymbol{C}^{n}\right)$ onto the $k$-th eigenspace.

The following is the analogue of Theorem 2.1 for the special Hermite expansions.

THEOREM 3.1. Let $A=\left\{a_{j}\right\}$ be a sequence of natural numbers such that $a_{0} \leq b$ and $a_{j+1}-a_{j} \leq b$. Let $f \in L^{2}\left(C^{n}\right)$ be such that $\|f\|_{2}=1$. Then we have the lower bound

$$
\int_{C^{n}}|z|^{2}|f(z)|^{2} d z \geq \frac{1}{8} b^{-2}
$$

whenever the condition

$$
\sum_{k \in A}\left\|f \times \varphi_{k}\right\|_{2}^{2} \leq \frac{1}{4} b^{-1}
$$

is satisfied. 
As in the case of Hermite expansions this theorem will be proved by reducing it to an inequality for Laguerre series. We will briefly indicate how this reduction is done.

The reduction is achieved by using a Hecke-Bochner type identity for the Weyl transform proved by Geller [2]. For each pair of non-negative integers $p$ and $q$, let $\mathcal{P}_{p, q}$ be the space of all polynomials $P$ in $z$ and $\bar{z}$ of the form

$$
P(z)=\sum_{|\alpha|=p} \sum_{|\beta|=q} c_{\alpha \beta} z^{\alpha} \bar{z}^{\beta}
$$

Let $\mathcal{H}_{p q}=\left\{P \in \mathcal{P}_{p q} ; \Delta P=0\right\}$, where $\Delta$ is the standard Laplacian on $\boldsymbol{C}^{n}$. Elements of $\mathcal{H}_{p q}$ are called bigraded solid harmonics. If $\mathcal{S}_{p q}$ are the restrictions of elements of $\mathcal{H}_{p q}$ to $S^{2 n-1}$, then $L^{2}\left(S^{2 n-1}\right)$ is the orthogonal direct sum of $\mathcal{S}_{p q}$.

Given a function $f$ on $\boldsymbol{C}^{n}$, consider the expansion

$$
f(r w)=\sum_{k=0}^{\infty} \sum_{p+q=k} f_{p q}(r w)
$$

where $z=r w, w \in S^{2 n-1}$ and $f_{p q} \in \mathcal{S}_{p q}$. The functions $f_{p q}$ can be represented in terms of certain unitary representations of the unitary group $U(n)$. For each $(p, q)$ there is an irreducible, unitary representation $\delta_{p q}$ of $U(n)$ on $\mathcal{S}_{p q}$. Then

$$
f_{p q}(z)=d(p, q) \int_{U(n)} \chi_{p q}(\sigma) f(\sigma \cdot z) d \sigma,
$$

where $\chi_{p q}$ is the character and $d(p, q)$ the degree of $\delta_{p q}$, see [4].

We first note that the special Hermite projections commute with the above decomposition. Indeed, using the above expression, recalling the definition of $f \times \varphi_{k}$ and noting that the symplectic form $\operatorname{Im}(z \cdot \bar{w})$ is preserved by $U(n)$, we get $f_{p q} \times \varphi_{k}(z)=\left(f \times \varphi_{k}\right)_{p q}(z)$. A result of Geller [2] says that elements of the form $f_{p q}$ have simple special Hermite expansions.

Proposition 3.2. Suppose $f \in L^{2}\left(\boldsymbol{C}^{n}\right)$ is of the form $f(z)=g(z) P(z)$, where $g$ is radial and $P \in \mathcal{H}_{p q}$. Then

$$
f \times \varphi_{k}(z)=c_{k} P(z) L_{k-p}^{n-1+p+q}\left(\frac{1}{2}|z|^{2}\right) e^{-(1 / 4)|z|^{2}},
$$

where $c_{k}=0$ for $k<p$ and for $k \geq p$

$$
c_{k}=\frac{(k-p) !(n-1) !}{(k+q+n-1) !} \int_{C^{n}} g(z) L_{k-p}^{n-1+p+q}\left(\frac{1}{2}|z|^{2}\right) e^{-(1 / 4)|z|^{2}}|z|^{2(p+q)} d z .
$$

A proof of this can be found in [2]. In [4] the above formula has been used in the study of twisted spherical means on $\boldsymbol{C}^{n}$. In view of the preceding remarks, in order to prove Theorem 3.1 it is enough to consider functions of the form $f(z)=g(z) P(z)$ with $g$ radial and $P \in \mathcal{H}_{p q}$. Then by the proposition we are reduced to proving an uncertainty inequality for Laguerre expansions. Theorem 3.1 thus follows from Theorem 5.1. 
4. Heisenberg group. Consider the Heisenberg group $H^{n}=\boldsymbol{C}^{n} \times \boldsymbol{R}$ equipped with the group law

$$
(z, t)(w, s)=\left(z+w, t+s+\frac{1}{2} \operatorname{Im}(z \cdot \bar{w})\right) .
$$

All the infinite dimensional irreducible unitary representations of $H^{n}$ are given, up to unitary equivalence, by the Schrodinger representations $\pi_{\lambda}, \lambda \in \boldsymbol{R}, \lambda \neq 0$. All these representations are realised on $L^{2}\left(\boldsymbol{R}^{n}\right)$; explicitly, they are given by

$$
\pi_{\lambda}(z, t) \varphi(\xi)=e^{i \lambda t} e^{i \lambda(x \cdot \xi+(1 / 2) x \cdot y)} \varphi(\xi+y)
$$

for $\varphi \in L^{2}\left(\boldsymbol{R}^{n}\right)$. The Fourier transform of an integrable function $f$ is defined to be the operator valued function

$$
\hat{f}(\lambda)=\int_{H^{n}} f(z, t) \pi_{\lambda}(z, t) d z d t .
$$

We refer to the monograph [7] for more about Heisenberg group and the Fourier transform.

A simple minded extension of the inequality (1.2) to the Heisenberg group set-up is the following. Let $\left\{a_{j}\right\}$ and $\left\{b_{j}\right\}$ be two sequences, $0<a_{j} \rightarrow \infty, 0>b_{j} \rightarrow-\infty, a_{j+1}-a_{j} \leq$ $b$ and $b_{j}-b_{j+1} \leq b$. Then, for $f \in L^{2}\left(H^{n}\right)$ normalised so that $\|f\|_{2}=1$, the condition

$$
\sum_{j=0}^{\infty}\left(\left\|\hat{f}\left(a_{j}\right)\right\|_{H S}^{2}+\left\|\hat{f}\left(b_{j}\right)\right\|_{H S}^{2}\right) \leq \frac{1-\varepsilon}{\sqrt{b}}
$$

for some $\varepsilon>0$ implies the lower bound

$$
\int_{H^{n}} t^{2}|f(z, t)|^{2} d z d t \geq 8 \frac{\varepsilon^{2}}{b^{2}} .
$$

The proof of this is almost the same as the proof of (1.2). In fact, an easy calculation shows that

$$
|\lambda|^{n}\|\hat{f}(\lambda)\|_{H S}^{2}=(2 \pi)^{n} \int_{C^{n}}|\tilde{f}(z, \lambda)|^{2} d z,
$$

where

$$
\tilde{f}(z, \lambda)=\int f(z, t) e^{i \lambda t} d t
$$

and hence the argument given in [5] proves the above estimate.

In this section we are interested in establishing a lower bound for the $L^{2}$ norm of $|z| f(z, t)$. In order to do this we are going to view the Fourier transform not as a function of the single variable $\lambda$ but as a function of $(\lambda, k), k=0,1,2, \ldots$. This can be compared with the point of view taken by Helgason [3] in considering the Euclidean Fourier transform $\hat{f}(\xi)$ as a function of $(\lambda, \omega)$, where $\xi=\lambda \omega, \omega \in S^{n-1}$. The best way to do this is to go to a bigger group, namely the Heisenberg motion group and look at the group Fourier transform there. 
The Heisenberg motion group denoted by $G^{n}$ is the semi-direct product of $U(n)$ and $H^{n}$ with the group law

$$
(\sigma, z, t)(\tau, w, s)=\left(\sigma \tau, z+\sigma w, t+s-\frac{1}{2} \operatorname{Im}(\sigma w \cdot \bar{z})\right) .
$$

Consider the set in $\boldsymbol{R}^{2}$ given by

$$
\{(\lambda,(2 k+n)|\lambda|) ; \lambda \neq 0, k=0,1,2, \ldots\},
$$

which is called the Heisenberg fan. To each point $(\lambda,(2 k+n)|\lambda|)$ on the Heisenberg fan there is an associated representation $\rho_{k}^{\lambda}$ of $G^{n}$. Let $\Phi_{\alpha}^{\lambda}(x)=|\lambda|^{n / 4} \Phi_{\alpha}\left(|\lambda|^{1 / 2} x\right)$, and define $E_{\alpha \beta}^{\lambda}(z, t)=\left(\pi_{\lambda}(z, t) \Phi_{\alpha}^{\lambda}, \Phi_{\beta}^{\lambda}\right)$. Let $\mathcal{H}_{k}^{\lambda}$ be the Hilbert space for which

$$
\left\{E_{\alpha \beta}^{\lambda} ; \alpha, \beta \in N^{n},|\alpha|=k\right\}
$$

is an orthonormal basis. $\rho_{k}^{\lambda}$ are realised on $\mathcal{H}_{k}^{\lambda}$ and the action is given by

$$
\rho_{k}^{\lambda}(\sigma, z, t) f(w, s)=f\left(\pi\left((\sigma, z, t)^{-1}\right)(w, s)\right),
$$

where $\pi(\sigma, z, t)(w, s)=(z, t)(\sigma w, s)$ is the action of $G^{n}$ on $H^{n}$. We refer to [7] for more about these representations.

Given a function $f$ on $G^{n}$, we can define $\rho_{k}^{\lambda}(f)$ as an operator on $\mathcal{H}_{k}^{\lambda}$. When $f \in$ $L^{1} \cap L^{2}\left(H^{n}\right)$, we can show that $\rho_{k}^{\lambda}(f)$ is a Hilbert-Schmidt operator and

$$
\left\|\rho_{k}^{\lambda}(f)\right\|_{H S}^{2}=|\lambda|^{n} \frac{k !(n-1) !}{(k+n-1) !} \int_{C^{n}}\left|f^{\lambda} *_{\lambda} \varphi_{k}^{\lambda}(w)\right|^{2} d w .
$$

Here $f^{\lambda}(z)=\tilde{f}(z, \lambda), \varphi_{k}^{\lambda}(w)=\varphi_{k}\left(|\lambda|^{1 / 2} w\right)$ and the $\lambda$-twisted convolution of $f^{\lambda}$ and $\varphi_{k}^{\lambda}$ is defined by

$$
f^{\lambda} *_{\lambda} \varphi_{k}^{\lambda}(z)=\int_{C^{n}} f^{\lambda}(z-w) \varphi_{k}^{\lambda}(w) e^{i(\lambda / 2) \operatorname{Im}(z \cdot \bar{w})} d w .
$$

Note that when $\lambda=1$, this is just the twisted convolution used in Section 3. In terms of $\rho_{k}^{\lambda}(f)$ we have the Plancherel theorem

$$
\|f\|_{2}^{2}=(2 \pi)^{-2 n-1} \int_{-\infty}^{\infty}\left(\sum_{k=0}^{\infty}\left\|\rho_{k}^{\lambda}(f)\right\|_{H S}^{2} \frac{(k+n-1) !}{k !(n-1) !}\right)|\lambda|^{n} d \lambda .
$$

We are now ready to state our uncertainty inequality.

THEOREM 4.1. Let $A=\left\{a_{j}\right\}$ be an increasing sequence of natural numbers such that $a_{0} \leq b, a_{j+1}-a_{j} \leq b$. Let $f \in L^{2}\left(H^{n}\right)$ with $\|f\|_{2}=1$. Then there are constants $C, c>0$ such that

whenever the inequality

$$
\int_{H^{n}}|z|^{2}|f(z, t)|^{2} d z d t \geq C b^{-2}
$$

$$
\int_{-\infty}^{\infty}\left(\sum_{k \in A}\left\|\rho_{k}^{\lambda}(f)\right\|_{H S}^{2} \frac{(k+n-1) !}{k !(n-1) !}\right)|\lambda|^{n} d \lambda \leq c b^{-1}
$$

is satisfied by the operators $\rho_{k}^{\lambda}(f)$. 
In view of the above observations we need to establish the following inequality:

$$
\begin{aligned}
& \int_{\boldsymbol{C}^{n}}|z|^{2}\left|f^{\lambda}(z)\right|^{2} d z \\
& \quad \geq C b^{-2} \int_{\boldsymbol{C}^{n}}\left|f^{\lambda}(z)\right|^{2} d z-b^{-1}|\lambda|^{n} \sum_{k \in A} \int_{\boldsymbol{C}^{n}}\left|f^{\lambda} *_{\lambda} \varphi_{k}(z)\right|^{2} d z .
\end{aligned}
$$

This is just an analogue of Theorem 3.1 and can be proved in a similar fashion. Indeed, we have an analogue of Proposition 3.2. When $f(z)=g(z) P(z)$ as in Proposition 3.2,

$$
f *_{\lambda} \varphi_{k}^{\lambda}(z)=c_{k}^{\lambda} P(z) L_{k-p}^{n-1+p+q}\left(\frac{1}{2}|\lambda||z|^{2}\right) e^{-(1 / 4)|\lambda||z|^{2}}
$$

where $c_{k}^{\lambda}$ are given by

$$
c_{k}^{\lambda}=\frac{(k-p) !(n-1) !}{(k+q+n-1) !} \int_{C^{n}} g(z) L_{k-p}^{n-1+p+q}\left(\frac{1}{2}|\lambda||z|^{2}\right) e^{-(1 / 4)|\lambda||z|^{2}}|z|^{2(p+q)} d z .
$$

It is now clear that as in the case of Theorem 3.1, everything boils down to the uncertainty inequality of Theorem 5.1 .

5. Laguerre expansions. Laguerre polynomials of type $\alpha>-1$ are defined by the equation

$$
e^{-t} t^{\alpha} L_{k}^{\alpha}(t)=\frac{1}{k !} \frac{d^{k}}{d t^{k}}\left(e^{-t} t^{k+\alpha}\right)
$$

for $t>0$ and $k=0,1,2, \ldots$. They satisfy the orthogonality relations

$$
\int_{0}^{\infty} L_{k}^{\alpha}\left(t^{2}\right) L_{j}^{\alpha}\left(t^{2}\right) e^{-t^{2}} t^{2 \alpha+1} d t=\frac{1}{2} \frac{\Gamma(k+\alpha+1)}{\Gamma(k+1)} \delta_{k_{j}} .
$$

Suitably normalised, they form an orthonormal basis for $L^{2}\left(\boldsymbol{R}^{+}, t^{2 \alpha+1} d t\right)$. In this section we establish the following result.

THEOREM 5.1. Let $A=\left\{a_{k}\right\}$ be an increasing sequence of natural numbers such that $a_{0} \leq b, a_{k+1}-a_{k} \leq b$. Let $\alpha \geq-1 / 2$ and let

$$
f(t)=\sum_{j=0}^{\infty} c_{j} L_{j}^{\alpha}\left(t^{2}\right) e^{-(1 / 2) t^{2}}
$$

be the Laguerre series of $f \in L^{2}\left(\boldsymbol{R}^{+}, t^{2 \alpha+1} d t\right)$. Then we have the inequality

$$
\int_{0}^{\infty}|f(t)|^{2} t^{2 \alpha+3} d t \geq \frac{1}{4} b^{-2} \int_{0}^{\infty}|f(t)|^{2} t^{2 \alpha+1} d t-\frac{1}{2} b^{-1} \sum_{j \in A}\left|c_{j}\right|^{2} \frac{\Gamma(j+\alpha+1)}{\Gamma(j+1)} .
$$

Once this theorem is established, Theorems 2.1,3.1 and 4.1 all follow immediately. Note that we get the lower bound

$$
\int_{0}^{\infty}|f(t)|^{2} t^{2 \alpha+3} d t \geq \frac{1}{8} b^{-2}
$$


whenever the Laguerre coefficients of $f$ satisfy the condition

$$
\sum_{j \in A}\left|c_{j}\right|^{2} \frac{\Gamma(j+\alpha+1)}{\Gamma(j+1)} \leq \frac{1}{4} b^{-1} .
$$

We now proceed to the proof of the above result.

If $f$ is given as in the theorem, its $L^{2}$ norm is given by

$$
\int_{0}^{\infty}|f(t)|^{2} t^{2 \alpha+1} d t=\frac{1}{2} \sum_{j=0}^{\infty}\left|c_{j}\right|^{2} \frac{\Gamma(j+\alpha+1)}{\Gamma(j+1)} .
$$

Let us write $A_{j}=c_{j}(\Gamma(j+\alpha+1) / \Gamma(j+1))^{1 / 2}$. By making use of the recursion relation (see [6])

$$
t L_{j}^{\alpha}(t)=(2 j+\alpha+1) L_{j}^{\alpha}(t)-(j+1) L_{j+1}^{\alpha}(t)-(j+\alpha) L_{j-1}^{\alpha}(t)
$$

valid for $j \geq 1$, we get

$$
\begin{aligned}
t^{2} f(t)= & c_{0} t^{2} L_{0}^{\alpha}\left(t^{2}\right) e^{-(1 / 2) t^{2}} \\
& +\sum_{j=1}^{\infty} c_{j}\left((2 j+\alpha+1) L_{j}^{\alpha}\left(t^{2}\right)-(j+1) L_{j+1}^{\alpha}\left(t^{2}\right)-(j+\alpha) L_{j-1}^{\alpha}\left(t^{2}\right)\right) e^{-(1 / 2) t^{2}} .
\end{aligned}
$$

Using the orthogonality properties of the Laguerre functions, we calculate that

$$
\begin{aligned}
\int_{0}^{\infty} t^{2}|f(t)|^{2} t^{2 \alpha+1} d t= & c_{0} \int_{0}^{\infty} L_{0}^{\alpha}\left(t^{2}\right) e^{-(1 / 2) t^{2}} t^{2} \bar{f}(t) t^{2 \alpha+1} d t \\
& +\frac{1}{2} \sum_{k=1}^{\infty}\left(\left|c_{k}\right|^{2} \frac{\Gamma(k+\alpha+1)}{\Gamma(k+1)}(2 k+\alpha+1)\right. \\
& \left.-c_{k} \bar{c}_{k+1} \frac{\Gamma(k+\alpha+2)}{\Gamma(k+2)}(k+1)-c_{k} \bar{c}_{k-1} \frac{\Gamma(k+\alpha)}{\Gamma(k)}(k+\alpha)\right) .
\end{aligned}
$$

Using the recursion relation once again and simplifying, we get

$$
\begin{aligned}
& \int_{0}^{\infty} t^{2}|f(t)|^{2} t^{2 \alpha+1} d t \\
& \quad=\frac{1}{2} \sum_{k=0}^{\infty}\left(\left|A_{k}\right|^{2}(2 k+\alpha+1)-2 \operatorname{Re}\left(A_{k} \bar{A}_{k+1}\right)(k+\alpha+1)^{1 / 2}(k+1)^{1 / 2}\right) .
\end{aligned}
$$

Since we also have the equality

$$
\int_{0}^{\infty}|f(t)|^{2} t^{2 \alpha+1} d t=\frac{1}{2} \sum_{k=0}^{\infty}\left|A_{k}\right|^{2},
$$


we need to establish

$$
\begin{aligned}
& \sum_{k=0}^{\infty}\left|A_{k}\right|^{2}(2 k+\alpha+1)-2 \operatorname{Re}\left(A_{k} \bar{A}_{k+1}\right)(k+\alpha+1)^{1 / 2}(k+1)^{1 / 2} \\
& \geq \frac{1}{4} b^{-2} \sum_{k=0}^{\infty}\left|A_{k}\right|^{2}-b^{-1} \sum_{k \in A}\left|A_{k}\right|^{2} .
\end{aligned}
$$

Writing $2 k+\alpha+1=(k+\alpha)+(k+1)$ and changing $k$ into $k+1$,

$$
\sum_{k=0}^{\infty}\left|A_{k}\right|^{2}(2 k+\alpha+1)=\sum_{k=0}^{\infty}\left|A_{k+1}\right|^{2}(k+\alpha+1)+\left|A_{k}\right|^{2}(k+1)+\alpha\left|A_{0}\right|^{2},
$$

and therefore the above inequality becomes

$$
\begin{gathered}
\sum_{j=0}^{\infty}\left|(j+\alpha+1)^{1 / 2} A_{j+1}-(j+1)^{1 / 2} A_{j}\right|^{2}+\alpha\left|A_{0}\right|^{2} \\
\geq \frac{1}{4} b^{-2} \sum_{j=0}^{\infty}\left|A_{j}\right|^{2}-b^{-1} \sum_{j \in A}\left|A_{j}\right|^{2}
\end{gathered}
$$

Case 1: $\alpha \geq 0$. Defining $\rho_{j}$ by the equation

$$
\left(1+\rho_{j}\right)=\left(1+\frac{\alpha}{j+1}\right)^{1 / 2}
$$

we see that

$$
(j+1) \rho_{j}=\frac{1}{2} \int_{0}^{\alpha}\left(1+\frac{s}{j+1}\right)^{-1 / 2} d s,
$$

is an increasing function in $j$ and converges to $\alpha / 2$ as $j \rightarrow \infty$. Further define a function $F(j)$ as

$$
F(j)=\alpha-j \rho_{j-1}-(j+1) \rho_{j},
$$

which is a decreasing function of $j$ and tends to 0 as $j \rightarrow \infty$. Let $J$ be the first integer such that $F(J) \leq b^{-2} / 4$ and let $L \leq J \leq M$ be integers in $A$ which are nearest to $J$.

Now we have

$$
\begin{aligned}
& \sum_{j=0}^{J-1}\left|(j+\alpha+1)^{1 / 2} A_{j+1}-(j+1)^{1 / 2} A_{j}\right|^{2} \\
& \quad=\sum_{j=0}^{J-1}\left((j+\alpha+1)\left|A_{j+1}\right|^{2}+(j+1)\left|A_{j}\right|^{2}-2(j+\alpha+1)^{1 / 2}(j+1)^{1 / 2} \operatorname{Re}\left(A_{j} \bar{A}_{j+1}\right)\right),
\end{aligned}
$$

which is bounded below by

$$
\sum_{j=0}^{J-1}(j+\alpha+1)\left|A_{j+1}\right|^{2}+(j+1)\left|A_{j}\right|^{2}-\left(\left|A_{j}\right|^{2}+\left|A_{j+1}\right|^{2}\right)(j+1)^{1 / 2}(j+\alpha+1)^{1 / 2} .
$$


This can be written as

$$
\begin{aligned}
& \sum_{j=0}^{J-1}\left((j+\alpha+1)^{1 / 2}-(j+1)^{1 / 2}\right)\left((j+\alpha+1)^{1 / 2}\left|A_{j+1}\right|^{2}-(j+1)^{1 / 2}\left|A_{j}\right|^{2}\right) \\
& \quad=\sum_{j=0}^{J-1}\left(1+\rho_{j}-1\right)\left(\left(1+\rho_{j}\right)\left|A_{j+1}\right|^{2}-\left|A_{j}\right|^{2}\right)(j+1) \\
& =\sum_{j=0}^{J-1}\left(\left(\rho_{j}^{2}+2 \rho_{j}\right)\left|A_{j+1}\right|^{2}-\rho_{j}\left(\left|A_{j+1}\right|^{2}+\left|A_{j}\right|^{2}\right)\right)(j+1) \\
& =\sum_{j=0}^{J-1}\left(\alpha\left|A_{j+1}\right|^{2}-\rho_{j}(j+1)\left(\left|A_{j+1}\right|^{2}+\left|A_{j}\right|^{2}\right)\right) \\
& =\sum_{j=0}^{J-1} F(j)\left|A_{j}\right|^{2}-\alpha\left|A_{0}\right|^{2}+\left(\alpha-J \rho_{J-1}\right)\left|A_{J}\right|^{2}
\end{aligned}
$$

Recalling the fact that $F(j) \geq(1 / 4) b^{-2}$ for $0 \leq j \leq J-1$ we have

$$
\begin{aligned}
& \sum_{j=0}^{J-1}\left|(j+\alpha+1)^{1 / 2} A_{j+1}-(j+1)^{1 / 2} A_{j}\right|^{2} \\
& \quad \geq \frac{1}{4} b^{-2} \sum_{j=0}^{J-1}\left|A_{j}\right|^{2}-\alpha\left|A_{0}\right|^{2}+\left(\alpha-J \rho_{J-1}\right)\left|A_{J}\right|^{2} .
\end{aligned}
$$

Next we estimate the sum taken from $j=J$ to $\infty$. Let $m$ and $l$ be natural numbers such that $l<m$. From the definition of $\rho_{j}$, we can see that

$$
\sum_{j=l}^{m-1}\left|(j+\alpha+1)^{1 / 2} A_{j+1}-(j+1)^{1 / 2} A_{j}\right|^{2}=\sum_{j=l}^{m-1}\left|\left(1+\rho_{j}\right) A_{j+1}-A_{j}\right|^{2}(j+1),
$$

which can be written as

$$
\sum_{j=l}^{m-1}\left(\left|A_{j+1}-A_{j}\right|^{2}(j+1)+\alpha\left|A_{j+1}\right|^{2}\right)+R
$$

where $R=-2 \sum_{j=l}^{m-1} \rho_{j}(j+1) \operatorname{Re}\left(A_{j} \bar{A}_{j+1}\right)$. Using the estimate

$$
|R| \leq \sum_{j=l}^{m-1} \rho_{j}(j+1)\left(\left|A_{j}\right|^{2}+\left|A_{j+1}\right|^{2}\right),
$$


we get

$$
\begin{aligned}
& \sum_{j=l}^{m-1}\left|(j+\alpha+1)^{1 / 2} A_{j+1}-(j+1)^{1 / 2} A_{j}\right|^{2} \\
& \quad \geq \sum_{j=l}^{m-1}\left[\left|A_{j+1}-A_{j}\right|^{2}(j+1)+\alpha\left|A_{j+1}\right|^{2}-\rho_{j}(j+1)\left(\left|A_{j+1}\right|^{2}+\left|A_{j}\right|^{2}\right)\right] \\
& \quad=\sum_{j=l}^{m-1}\left|A_{j+1}-A_{j}\right|^{2}(j+1)+E
\end{aligned}
$$

where the error term $E$ is given by

$$
E=\sum_{j=l}^{m-1}\left(\alpha\left|A_{j+1}\right|^{2}-\rho_{j}(j+1)\left(\left|A_{j+1}\right|^{2}+\left|A_{j}\right|^{2}\right)\right) .
$$

It is easy to get a lower bound for the main term in (5.7). Since

$$
A_{k}=A_{l}+\sum_{j=l}^{k-1}\left(A_{j+1}-A_{j}\right)
$$

for $l \leq k \leq m-1$, we get the estimate

$$
\frac{1}{2}\left|A_{k}\right|^{2} \leq\left|A_{l}\right|^{2}+\left(\sum_{j=l}^{m-1}\left|A_{j+1}-A_{j}\right|\right)^{2} .
$$

Applying Cauchy-Schwarz inequality and noting that $m-l \leq b$, we get the estimate

$$
\frac{1}{2} \sum_{k=l}^{m-1}\left|A_{k}\right|^{2} \leq b\left|A_{l}\right|^{2}+b^{2} \sum_{j=l}^{m-1}\left|A_{j+1}-A_{j}\right|^{2}
$$

Thus we have

$$
\sum_{j=l}^{m-1}\left|A_{j+1}-A_{j}\right|^{2}(j+1) \geq \frac{1}{2} b^{-2} \sum_{j=l}^{m-1}\left|A_{j}\right|^{2}-b^{-1}\left|A_{l}\right|^{2} .
$$

In order to estimate the error term in (5.7) we observe that

$$
\begin{aligned}
E & =\sum_{j=l}^{m-1} F(j)\left|A_{j}\right|^{2}-\left(\alpha-l \rho_{l-1}\right)\left|A_{l}\right|^{2}+\left(\alpha-m \rho_{m-1}\right)\left|A_{m}\right|^{2} \\
& \geq-\left(\alpha-l \rho_{l-1}\right)\left|A_{l}\right|^{2}+\left(\alpha-m \rho_{m-1}\right)\left|A_{m}\right|^{2}
\end{aligned}
$$


recalling the fact that $F(j)$ is non-negative. Combining this with (5.8), we have

$$
\begin{aligned}
& \sum_{j=l}^{m-1}\left|(j+\alpha+1)^{1 / 2} A_{j+1}-(j+1)^{1 / 2} A_{j}\right|^{2} \\
& \quad \geq \frac{1}{2} b^{-2} \sum_{j=l}^{m-1}\left|A_{j}\right|^{2}-b^{-1}\left|A_{l}\right|^{2}-\left(\alpha-l \rho_{l-1}\right)\left|A_{l}\right|^{2}+\left(\alpha-m \rho_{m-1}\right)\left|A_{m}\right|^{2}
\end{aligned}
$$

which leads to the estimate

$$
\begin{aligned}
& \sum_{j=J}^{\infty}\left|(j+\alpha+1)^{1 / 2} A_{j+1}-(j+1)^{1 / 2} A_{j}\right|^{2} \\
& \quad \geq \frac{1}{4} b^{-2} \sum_{j=J}^{\infty}\left|A_{j}\right|^{2}-b^{-1} \sum_{M \leq j \in A}\left|A_{j}\right|^{2}-\left(\alpha-J \rho_{J-1}\right)\left|A_{J}\right|^{2}
\end{aligned}
$$

From (5.6) and (5.10) we have

$$
\begin{aligned}
& \sum_{j=0}^{\infty}\left|(j+\alpha+1)^{1 / 2} A_{j+1}-(j+1)^{1 / 2} A_{j}\right|^{2} \\
& \quad \geq \frac{1}{4} b^{-2} \sum_{j=0}^{\infty}\left|A_{j}\right|^{2}-b^{-1} \sum_{j \in A}\left|A_{j}\right|^{2}-\alpha\left|A_{0}\right|^{2}
\end{aligned}
$$

which proves the estimate (5.4), if $\alpha \geq 0$.

Case $2:-1 / 2 \leq \alpha<0$. Defining $\rho_{j}$ by the equation

$$
\left(1+\rho_{j}\right)=\left(1-\frac{\alpha}{j+\alpha+1}\right)^{1 / 2}
$$

we can see that,

$$
(j+\alpha+1) \rho_{j}=\frac{1}{2} \int_{0}^{-\alpha}\left(1+\frac{s}{j+\alpha+1}\right)^{-1 / 2} d s
$$

is an increasing function in $j$ and converges to $-\alpha / 2$ as $j \rightarrow \infty$. Also define a function $F(j)$ as

$$
F(j)=-\alpha-(j+\alpha) \rho_{j-1}-(j+\alpha+1) \rho_{j}
$$

which is a decreasing function of $j$ and tends to 0 as $j \rightarrow \infty$. As in the case 1 , choose $L, J$ and $M$ such that $F(J) \leq(1 / 4) b^{-2}$. 
Arguing as in Case 1 we have

$$
\begin{aligned}
\sum_{j=0}^{J-1} \mid & (j+\alpha+1)^{1 / 2} A_{j+1}-\left.(j+1)^{1 / 2} A_{j}\right|^{2} \\
\geq & \sum_{j=0}^{J-1}\left(1-\left(1+\rho_{j}\right)\right)\left(\left|A_{j+1}\right|^{2}-\left(1+\rho_{j}\right)\left|A_{j}\right|^{2}\right)(j+\alpha+1) \\
= & \sum_{j=0}^{J-1}\left(\left(\rho_{j}^{2}+2 \rho_{j}\right)\left|A_{j}\right|^{2}-\rho_{j}\left(\left|A_{j+1}\right|^{2}+\left|A_{j}\right|^{2}\right)\right)(j+\alpha+1) \\
= & \sum_{j=0}^{J-1}\left(-\alpha\left|A_{j}\right|^{2}-\rho_{j}(j+\alpha+1)\left(\left|A_{j+1}\right|^{2}+\left|A_{j}\right|^{2}\right)\right) \\
= & \sum_{j=0}^{J-1} F(j)\left|A_{j}\right|^{2}-\alpha\left|A_{0}\right|^{2}-(\alpha+J) \rho_{J-1}\left|A_{J}\right|^{2} \\
\geq & \frac{1}{4} b^{-2} \sum_{j=0}^{J-1}\left|A_{j}\right|^{2}-\alpha\left|A_{0}\right|^{2}-(\alpha+J) \rho_{J-1}\left|A_{J}\right|^{2} .
\end{aligned}
$$

noting that $\rho_{-1}=-1$.

For estimating the sum taken from $J$ to $\infty$ we consider the sum over blocks, $l \leq j<m$ where $l, m \in A$, as in Case 1 .

We have

$$
\sum_{j=l}^{m-1}\left|(j+\alpha+1)^{1 / 2} A_{j+1}-(j+1)^{1 / 2} A_{j}\right|^{2}=\sum_{j=l}^{m-1}\left|A_{j+1}-\left(1+\rho_{j}\right) A_{j}\right|^{2}(j+\alpha+1),
$$

which can be written as

$$
\sum_{j=l}^{m-1}\left|A_{j+1}-A_{j}\right|(j+\alpha+1)+R
$$

where $R=\sum_{j=l}^{m-1}\left(\left(2 \rho_{j}+\rho_{j}^{2}\right)\left|A_{j}\right|^{2}-2 \rho_{j} \operatorname{Re}\left(A_{j} \bar{A}_{j+1}\right)\right)(j+\alpha+1)$.

The main term in (5.12) can be estimated as in Case 1 and the error term $R$ can be bounded below as follows.

$$
\begin{aligned}
R & \geq \sum_{j=l}^{m-1}\left(\left(2 \rho_{j}+\rho_{j}^{2}\right)\left|A_{j}\right|^{2}-\rho_{j}\left(\left|A_{j}\right|^{2}+\left|A_{j+1}\right|^{2}\right)\right)(j+\alpha+1) \\
& =\sum_{j=l}^{m-1}\left(-\alpha\left|A_{j}\right|^{2}-\rho_{j}(j+\alpha+1)\left(\left|A_{j}\right|^{2}+\left|A_{j+1}\right|^{2}\right)\right)
\end{aligned}
$$




$$
\begin{aligned}
& =\sum_{j=l}^{m-1} F(j)\left|A_{j}\right|^{2}+(\alpha+l) \rho_{l-1}\left|A_{l}\right|^{2}-(\alpha+m) \rho_{m-1}\left|A_{m}\right|^{2} \\
& \geq(\alpha+l) \rho_{l-1}\left|A_{l}\right|^{2}-(\alpha+m) \rho_{m-1}\left|A_{m}\right|^{2},
\end{aligned}
$$

since $F(j)$ is non-negative.

Following the steps as in Case 1, we get

$$
\begin{aligned}
& \sum_{j=J}^{\infty}\left|(j+\alpha+1)^{1 / 2} A_{j+1}-(j+1)^{1 / 2} A_{j}\right|^{2} \\
& \quad \geq \frac{1}{4} b^{-2} \sum_{j=J}^{\infty}\left|A_{j}\right|^{2}-b^{-1} \sum_{M \leq j \in A}\left|A_{j}\right|^{2}+(\alpha+J) \rho_{J-1}\left|A_{J}\right|^{2} .
\end{aligned}
$$

Combining (5.11) and (5.13), we have

$$
\begin{aligned}
& \sum_{j=0}^{\infty}\left|(j+\alpha+1)^{1 / 2} A_{j+1}-(j+1)^{1 / 2} A_{j}\right|^{2} \\
& \geq \frac{1}{4} b^{-2} \sum_{j=0}^{\infty}\left|A_{j}\right|^{2}-b^{-1} \sum_{j \in A}\left|A_{j}\right|^{2}-\alpha\left|A_{0}\right|^{2},
\end{aligned}
$$

which proves the estimate (5.4), for the case $-1 / 2 \leq \alpha<0$. Hence Theorem 5.1 is established.

\section{REFERENCES}

[ 1 ] G. B. Folland and A. Sitaram, The uncertainty principle: A mathematical survey, J. Fourier Anal. Appl. 3 (1997), 207-238.

[2] D. Geller, Spherical harmonics, the Weyl transform and the Fourier transform on the Heisenberg group, Canad. J. Math. 36 (1984), 615-684.

[3] S. Helgason, Geometric analysis on symmetric spaces, Math. Surveys Monogr. 39, Amer. Math. Soc., Providence, RI, 1994.

[ 4 ] E. K. NARAYAnAN And S. Thangavelu, Injectivity sets for spherical means on the Heisenberg group, J. Math. Anal. Appl. 263 (2001), 565-579.

[ 5 ] R. Strichartz, Uncertainty principles in harmonic analysis, J. Funct. Anal. 84 (1989), 97-114.

[6] S. ThangaVelu, Lectures on Hermite and Laguerre expansions, Math. Notes 42, Princeton university press, Princeton, NJ, 1993.

[ 7 ] S. Thangavelu, Harmonic analysis on the Heisenberg group, Progr. Math. 159, Birkhäuser Boston, Inc., Boston, MA, 1998.

RESEARCH SCHOLAR

DEPARTMENT OF MATHEMATICS

CALICUT UNIVERSITY

MALAPPURAM-673635

INDIA

E-mail address: smithacnair@yahoo.com
STATISTICS AND MATHEMATICS UNIT

InDian STATISTICAL Institute

8TH Mile, Mysore RoAD

BANGALORE-560059

INDIA

E-mail address: veluma@isibang.ac.in 